\title{
RESONANCE FAR-FIELD ACCELERATING STRUCTURES AT SHORT WAVELENGTHS
}

\author{
A.V. Smirnov \\ Russian Research Center «Kurchatov Institute», 123182 Moscow, Russian Federation
}

\begin{abstract}
Resonance far-field structures capable to provide high gradient continuos acceleration at millimeter and micron wavelengths are considered. Among such structures are periodically striped open waveguide and oversized waveguide having periodic small perturbations. Main parameters of the structure composed by flat mirrors are estimated on the base of rigorous analytical solution for the eigenmodes.

Some modifications of diffraction-dominated structures based on open waveguides and resonators are proposed.
\end{abstract}

\section{INTRODUCTION}

In the previous paper [1] a concept was proposed for laser resonance acceleration of relativistic charged particles. It is based on a resonance interaction of a straightforward charged particle beam interacting with electromagnetic beam having periodic change of wave vector. Closed waveguide having periodical deformation was considered as the accelerating structure. It was shown earlier, that such an overmoded rectangular [2] or circular [3] waveguide can be excited effectively (with dominant single mode) by a properly focused laser beam having linear polarization. However, the stability of the hybrid $\mathrm{EH}_{11}$ mode to the transformation into parasitic higher modes requires further consideration.

In this paper we consider another realization of the principle above based on an open periodic waveguide. It can provide stable propagation of the lowest mode due to diffraction resulting in widely-spaced spectrum of eigenmodes.

\section{EIGENMODES OF THE PERIODICAL OPEN MIRROR WAVEGUIDE}

The open waveguide (or open periodical resonator) composed by chain of mirrors with period $\lambda_{w}$. For simplicity of consideration we assumed here rectangular flat mirrors with dimensions $2 \mathrm{a} \times 2 \mathrm{~b}$. The principal scheme is shown in the Fig. 1. To consider the fields of the open mirror waveguide we solve first the problem for the equivalent open resonator with rectangular mirrors having dimensions $2 a_{1} \times 2 b$ (see Fig. 2). The e.m. fields of the resonator with rectangular mirrors are found by Vainshtein [4] in terms of eigenmodes for Hertz vector potential $\vec{\Pi}$ at $\lambda_{\mathrm{s}}<<\mathrm{a}, \mathrm{b}$, where $\lambda_{\mathrm{s}}=2 \pi \mathrm{c} / \omega$ is laser wavelength. The vector potential $\vec{\Pi}$ is defined through the potential function $\Phi$, that satisfies to a Helmholtz wave equation: $\Delta \Phi+\mathrm{k}^{2} \Phi=0$.

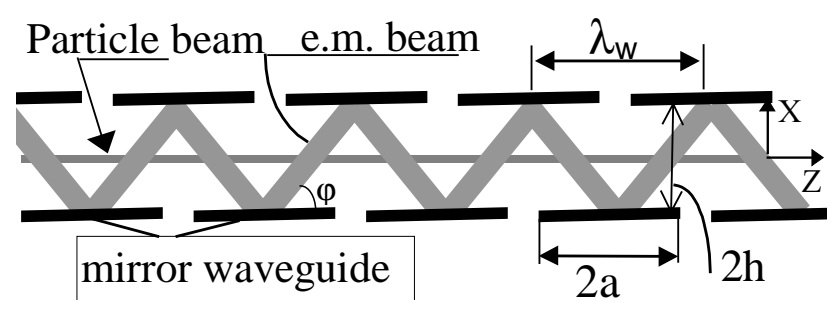

Fig. 1. Schematic drawing of an accelerator with a «wiggling» field propagating in an open mirror waveguide.

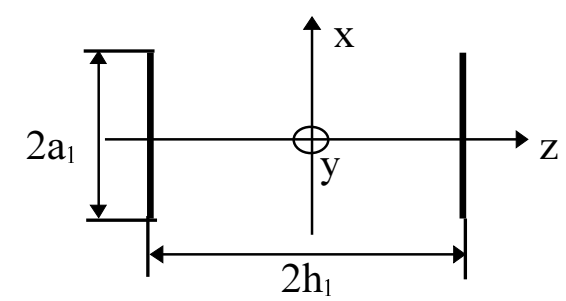

Fig. 2. Equivalent open resonator with rectangular mirrors.

If the fields in the resonator are produced by plain waves propagating with small angles to the axis OZ, the fields of corresponding TE modes can be expressed in the following form:

$$
\left(\begin{array}{l}
E \\
\vec{H}
\end{array}\right)=\operatorname{Re}\left(\begin{array}{l}
i k r o t \vec{\Pi}_{\mathrm{TE}} \\
\operatorname{grad} \operatorname{div} \vec{\Pi}_{\mathrm{TE}}+\mathrm{k}^{2} \vec{\Pi}_{\mathrm{TE}}
\end{array}\right) \mathrm{e}^{-i \omega \mathrm{t},}
$$

where $\vec{\Pi}_{\mathrm{TE}}=\Phi \overrightarrow{\mathrm{e}}_{\mathrm{y}}, \mathrm{k}=\omega / \mathrm{c}$,

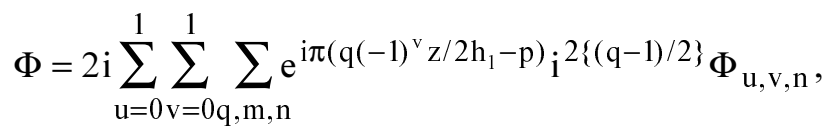

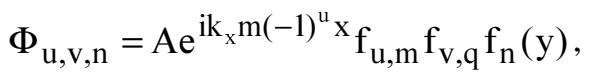

$$
\begin{aligned}
& \mathrm{f}_{\mathrm{u}, \mathrm{m}}=-\left(1-\mathrm{u}-(-1)^{\mathrm{m}} \mathrm{u}\right)(-1)^{[(\mathrm{n}-1) / 2]} / 2 \mathrm{i}^{\mathrm{n}+1}, \\
& f_{n}(y)=\left\{\begin{array}{l}
\cos \left(n k_{y} y\right), n=1,3,5 \ldots \\
\sin \left(n k_{y} y\right), n=2,4,6 \ldots
\end{array}, \quad M_{a, b}=\sqrt{\frac{2 k}{h_{1}}\left(a_{1}^{2} b^{2}\right)},\right. \\
& \mathrm{k}_{\mathrm{a}, \mathrm{b}}=\frac{\pi / 2}{\left(\mathrm{a}_{1}, \mathrm{~b}\right)\left(1+\eta(1+\mathrm{i}) / \mathrm{M}_{\mathrm{a}, \mathrm{b}}\right)}, \quad \eta=-\zeta(-0.5) \pi^{-0.5} \approx 0.824,
\end{aligned}
$$


$\mathrm{p}_{\mathrm{a}, \mathrm{b}}=\frac{(\pi / 4)\left(\mathrm{m}^{2}, \mathrm{n}^{2}\right)}{\left(\mathrm{M}_{\mathrm{a}, \mathrm{b}}+\eta(1+\mathrm{i})\right)^{2}}, \quad \mathrm{p}=\mathrm{p}_{\mathrm{a}}+\mathrm{p}_{\mathrm{b}}, \zeta(\mathrm{z})$ is Riehmann's

Zeta function.

To find the potential function for the waveguide it is necessary to make a transition from the standing wave to the traveling one, to make a transformation from local frame xoz (see Fig. 2 corresponding to each half a period) to the waveguide frame XOZ (Fig. 1) and to impose Flouqet condition of periodicity. The transformation is valid under the following conditions:

$\lambda_{\mathrm{s}}<<\lambda_{\mathrm{w}} \pi \mathrm{p}, '<<1$ and $\mathrm{h}_{1}=\mathrm{h} / \sin \varphi, \mathrm{a}_{1}=\mathrm{a} \sin \varphi$,

where $\mathrm{p}=\mathrm{p}$ '-ip',

After Fourier expanding of the potential function on the period $\lambda_{\mathrm{w}}$ one can derive:

$$
\Phi=A e^{i k_{Z} Z} \sum_{q_{w}} C_{q_{w}} e^{i q_{w} k_{w} Z}, C_{q}=\sum_{u, m, n} f_{n}(y) f_{u, m} B_{u, m, n}^{(q)},
$$

where $\mathrm{k}_{\mathrm{z}}=\left(\mathrm{k}-2 \mathrm{k}_{\mathrm{w}} \mathrm{p}^{\prime} \cos \varphi\right) \cos \varphi-\mathrm{ik}_{\mathrm{w}} \mathrm{p}^{\prime}, \mathrm{q}_{\mathrm{w}}=0,1,2,3 \ldots$,

$$
\begin{aligned}
& \mathrm{B}_{\mathrm{u}, \mathrm{m}, \mathrm{n}}^{(\mathrm{q})}=\sum_{ \pm}\left\{\begin{array}{l}
\mathrm{e}^{\mathrm{i}\left(\mathrm{k}_{\mathrm{cu}} \mathrm{X}-\mathrm{p}^{\prime} 3 \pi / 2\right)} \mathrm{S}\left(\frac{\pi}{2}\left(\frac{\mathrm{k}_{\mathrm{su}}}{\mathrm{k}_{\mathrm{w}}} \mp \mathrm{q}_{\mathrm{w}}\right)\right) \times \\
\exp \left[ \pm \mathrm{i}\left(\mathrm{k}_{\mathrm{Z}}^{\prime} \mathrm{Xtg} \varphi+\frac{\pi}{2}\left(\mathrm{p}^{\prime}+\frac{\mathrm{k}_{\mathrm{su}}}{\mathrm{k}_{\mathrm{w}}} \mp \mathrm{q}_{\mathrm{w}}\right)\right)\right]
\end{array}\right\}, \\
& \mathrm{k}_{\mathrm{cu}}=\mathrm{k}_{\mathrm{am}}(-1)^{\mathrm{u}+1} \cos \varphi, \quad \mathrm{k}_{\mathrm{su}}=\mathrm{k}_{\mathrm{am}}(-1)^{\mathrm{u}+1} \sin \varphi, \\
& \mathrm{S}(\mathrm{x})=\sin (\mathrm{x}) / \mathrm{x}, \mathrm{k}_{\mathrm{w}}=2 \pi / \lambda_{\mathrm{w}} \text {. }
\end{aligned}
$$

\section{BASIC RELATIONSHIPS}

One can see from (3), that the Fourier coefficient $\mathrm{C}_{\mathrm{q}_{\mathrm{w}}}$ is a function of coordinate $\mathrm{X}$ due to wave front inclination. With taking into account condition $\lambda_{\mathrm{s}}<<\mathrm{a}, \mathrm{b}$ the dependency is weak at small glancing angles $\varphi<<1$ :

$\left(\mathrm{k}_{\mathrm{z}}{ }^{\prime} \mathrm{X} \operatorname{tg} \varphi\right)^{2}<<1$, or $(\varphi \mathrm{Xk})^{2}<<1$

If the angle $\varphi$ is small, substantial contribution in $\Phi$ give the following terms: $\mathrm{q}_{\mathrm{w}}=0, \mathrm{q}_{\mathrm{w}}=$ odd. We consider below only resonance acceleration, i.e. $\mathrm{q}_{\mathrm{w}}=1,3,5, \ldots$.

Phase velocity related to the speed of light follows simply from (3):

$$
\beta_{\mathrm{ph}}=\left(\mathrm{q}_{\mathrm{w}} \frac{\mathrm{k}_{\mathrm{w}}}{\mathrm{k}}+\left(1-2 \frac{\mathrm{k}_{\mathrm{w}}}{\mathrm{k}} \mathrm{p}^{\prime} \cos \varphi\right) \cos \varphi\right)^{-1} .
$$

Then for $\gamma>>1$ we have the following condition of resonance:

$$
\frac{\lambda_{\mathrm{w}}}{\lambda_{\mathrm{s}}}=\frac{\mathrm{q}_{\mathrm{w}}+4 \mathrm{p}^{\prime}}{2 \sin ^{2}(\varphi / 2)+\gamma^{-2} / 2}
$$

One can obtain from (5) for $\gamma^{2} \varphi^{2}>>1, \varphi<<1$ and $M_{a}$, $\mathrm{M}_{\mathrm{b}}>>1$ :

$$
\lambda_{\mathrm{w}} / \lambda_{\mathrm{s}} \approx 2 \mathrm{q}_{\mathrm{w}} / \varphi^{2}
$$

Note, that (6) is equivalent to analogous condition (2) in [1] when $\varphi \rightarrow \pi \mathrm{r}_{\mathrm{w}} \sqrt{ } 2 / \lambda_{\mathrm{w}}<<1$. From (6) one can estimate the tolerance on the most critical parameter $\varphi: \Delta \varphi / \varphi<<1 / 2 \mathrm{q}_{\mathrm{w}}$.

For the practical situation, when $a-\lambda_{w} / 4<<a$, we have from (6) and (2) the following important rule:

$\mathrm{N}_{\mathrm{fx}}=\mathrm{q}_{\mathrm{w}} / 2$,

where $\mathrm{N}_{\mathrm{fx}}=\mathrm{M}_{\mathrm{a}}{ }^{2} / 8 \pi$ is the Fresnel number for XOZ plane.
If $M_{a}, M_{b} \gg 1$ and waist of the incident laser beam is matched with the mirror dimensions $a_{1}, b$ (analogously to $[2,3]$ ), we can assume, that the dominant propagating mode is $m=1, n=1$. Then the power flow through the waveguide is:

$$
\mathrm{P}=\mathrm{P}_{0} \mathrm{e}^{-2 \mathrm{k}_{\mathrm{Z}}^{\prime \prime} \mathrm{Z}} \text {, where }
$$

$\mathrm{P}_{0}=|\mathrm{A}|^{2}\left(\mathrm{k}^{2}-\frac{\mathrm{k}_{\mathrm{a}}^{2}+\mathrm{k}_{\mathrm{b}}^{2}}{2}\right) \mathrm{a}_{1} \mathrm{~b} \frac{\mathrm{k}^{2}-\mathrm{k}_{\mathrm{b}}^{2}}{2 \mathrm{Z}_{0}}, \mathrm{k}_{\mathrm{Z}}^{\prime \prime}=\mathrm{k}_{\mathrm{w}} \mathrm{p}^{\prime \prime}, \mathrm{Z}_{\mathrm{o}}=120 \pi \Omega$.

For the lowest dominant mode we obtain from $(1,3)$ the following expression for the accelerating field amplitude:

$$
\mathrm{E}_{\mathrm{a}}=-\mathrm{iAB} \mathrm{B}_{1,1,1}^{(\mathrm{q})} \mathrm{kk}_{\mathrm{Z}}^{\prime} \operatorname{tg} \varphi \cos \left(\mathrm{k}_{\mathrm{s} 1}\right) \cos \left(\mathrm{k}_{\mathrm{Z}}^{\prime} \operatorname{tg} \varphi \Delta \mathrm{X}\right)
$$

where $\Delta X=X-X_{0}, \quad X_{O}=\frac{\pi\left(1-p^{\prime}\right)}{2 k_{Z}^{\prime} \operatorname{tg} \varphi} \approx \frac{1}{4} \sqrt{\frac{\lambda_{W} \lambda_{s}}{2 q_{w}}}$ defines the optimal transverse position for the particle beam centroid.

In order to remove the details of the specific accelerator mechanism let us define the coupling strength $g_{c}$ as the ratio between the local acceleration gradient $\mathrm{E}_{\mathrm{a}}$ and vacuum electric field $\sqrt{2 \mathrm{Z}_{0} \mathrm{P}_{0} / \mathrm{S}_{0}}$ of the focused laser beam (here $S_{0} \approx 4 \mathrm{a}_{1} \mathrm{~b}$ ). Under the conditions above we have from $(7,8)$ :

$$
\mathrm{g}_{\mathrm{c}} \approx 4 \varphi / \mathrm{q}_{\mathrm{w}}=4 \sqrt{2 \lambda_{\mathrm{s}} / \mathrm{q}_{\mathrm{w}} \lambda_{\mathrm{w}}} .
$$

Note, for our scheme $\mathrm{g}_{\mathrm{c}}$ is equal approximately to $\mathrm{E}_{\mathrm{a}} / \mathrm{E}_{\mathrm{e}}$ defining the ratio between accelerating field and surface field.

Power attenuation length $\mathrm{L}_{\text {att }}$ can be defined from (7) provided ohmic losses are negligible:

$$
\mathrm{L}_{\mathrm{att}}=1 / 2 \mathrm{k}_{\mathrm{w}} \mathrm{p}^{\prime \prime} \approx \frac{\lambda_{\mathrm{w}}}{2 \pi^{2} \eta \mathrm{M}_{\mathrm{a}}^{3}+\mathrm{M}_{\mathrm{b}}^{3}} .
$$

\section{PERFORMANCE ESTIMATIONS}

It is seen from (10), that power losses are proportional to $\lambda_{\mathrm{w}}{ }^{-1} \mathrm{q}_{\mathrm{w}}{ }^{-3 / 2}$, whereas the coupling strength scales as

$\lambda_{\mathrm{w}}^{-1} \mathrm{q}_{\mathrm{w}}^{-1}$. Hence, to use the advantage of long resonance acceleration we should choose higher harmonics. However, as $\mathrm{q}_{\mathrm{w}}$ increases tolerances becomes more stringent. Estimated performance parameters of the scheme are presented in the Table 1 for $a_{1} \approx b / 3, a \approx \lambda_{w} / 4$, wavelength $\lambda_{\mathrm{s}}=10 \mu \mathrm{m}$ and surface field $\mathrm{E}_{\mathrm{e}}=5 \mathrm{GV} / \mathrm{m}$.

\section{DISCUSSION}

Since the angle $\varphi$ is small for short wavelengths, ohmic losses of the TE modes can be considerably reduced with the help of special corrugations made on the mirror surface (having period $\leq \lambda_{\mathrm{s}} / 2$ and depth $(0.2 \div 0.5) \lambda_{\mathrm{s}}$ ) or proper dielectric coating (see [2,5]). Besides, the radiation losses defined in (10) on the basis of diffraction in resonator (Fig. 2) for waveguide are actually less due to interference effect [4]. The diffraction losses can be reduced further by enhancement of both the effect with 
proper choose of the relationships between $\lambda_{\mathrm{s},} \lambda_{\mathrm{w},} \mathrm{a}, \varphi$ and figure of merit $\mathrm{Q}$ for single cell due to mirror curvature optimization.

In general case input coupling can give a combination of TE and TM mode [3]. Note, for the TM mode one can derive from (3), that coupling strength is less $\left(g_{c} \approx 4 \varphi^{2} / q_{w}\right)$ and $\mathrm{X}_{\mathrm{o}}=0$. To solve this multi-mode problem further study is necessary.

The main disadvantage of the scheme compared to an Open Iris Loaded Waveguide (OILS, [6]) is dependence of the acceleration rate on the particle transverse coordinate (3). This imposes rather stringent condition for the both particle beam radius and beam alignment tolerance that practically should not exceed several wavelengths (see (4)).

Table 1. Performance parameters for the rectangular mirror waveguide.

\begin{tabular}{|l|l|l|}
\hline $\mathrm{q}_{\mathrm{w}}$ & 3 & 5 \\
\hline$\lambda_{\mathrm{w}}, \mathrm{cm}$ & 1.2 & 4 \\
\hline$\varphi, \mathrm{mrad}$ & 70 & 50 \\
\hline $2 \mathrm{~h}, \mathrm{~mm}$ & 0.43 & 1.0 \\
\hline $\mathrm{g}_{\mathrm{c}}$ & 0.06 & 0.025 \\
\hline $\mathrm{M}_{\mathrm{a}}$ & 6.1 & 7.9 \\
\hline $\mathrm{L}_{\mathrm{att}}, \mathrm{cm}$ & 12.2 & 80 \\
\hline $\mathrm{I}_{\mathrm{e}}=\mathrm{P}_{0} / \mathrm{S}_{0}, \mathrm{~W} / \mathrm{cm}^{2}$ & $4.5 \cdot 10^{16}$ & $1.3 \cdot 10^{17}$ \\
\hline $\mathrm{P}_{0}, \mathrm{~W}$ & $2.5 \cdot 10^{10}$ & $4 \cdot 10^{11}$ \\
\hline $\mathrm{R}_{\mathrm{s}}, \mathrm{M} \Omega / \mathrm{m}$ & 0.6 & 0.12 \\
\hline $\mathrm{Q}$ & $7.6 \cdot 10^{4}$ & $5 \cdot 10^{5}$ \\
\hline $\mathrm{E}_{\mathrm{a}}, \mathrm{GeV} / \mathrm{m}$ & 0.35 & 0.25 \\
\hline
\end{tabular}

\section{SOME VARIANTS}

To reduce the diffraction losses limiting the acceleration section length one can use cylindrical or spherical mirrors (see Fig. 3).

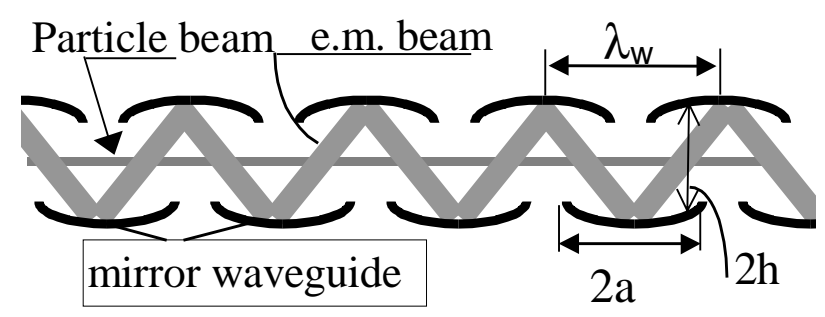

Fig. 3. Accelerating waveguide with reduced losses.

One can provide a resonance mechanism in OILW by means of special sections, where the interaction between particle beam and field is negligible (see Fig. 4). In this sections the propagating mode is converted into A-mode of spheroidal cavity [4]. In the scheme $\lambda_{w} / 2$ lies between $\mathrm{L}_{\mathrm{s}} / 2$ and $\mathrm{L}_{\mathrm{s}}$ (slippage length). An average acceleration rate is less then that local value for OILW (up to twofold).

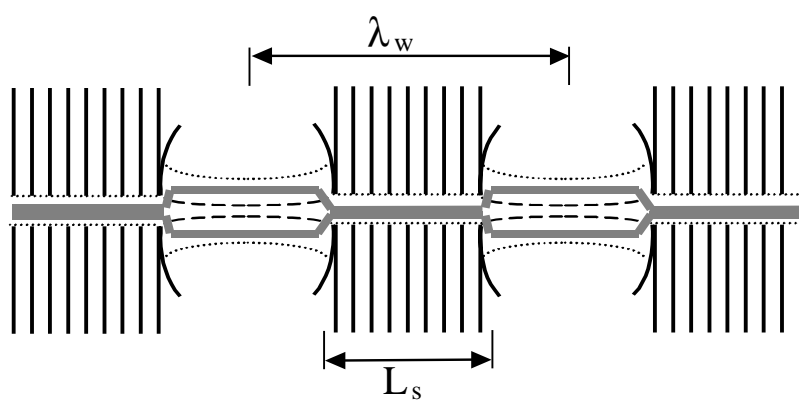

Fig. 4. Modified OILW to provide resonance acceleration. Gray thick lines show flow of primary power. Dotted lines show caustics.

\section{CONCLUSION}

One can outline the following attractable features of the acceleration scheme considered:

- Relative simplicity for manufacturing of the waveguide composed by flat mirrors and suitability for acceleration of flat beams.

- The final energy gain is limited by attenuation length rather than slippage length, because it is resonance acceleration.

- Tapering is not necessary to provide synchronism between relativistic particles and accelerating harmonic.

- Axicon scheme is not required to couple the laser power in the structure.

- Synchrotron radiation losses are very low due to low equivalent undulator strength $\mathrm{K}_{\mathrm{w}} \leq 1[1]$.

\section{ACKNOLEDGMENTS}

The author would like to thank Dr. David Yu for deep interest and many helpful discussions.

\section{REFERENCES}

[1] A.V. Smirnov, Far-Field Acceleration Scheme, Proc. of the $6^{\text {th }}$ European Particle Acceleration Conf., Stockholm, 22-26 June, 1998, p. 824.

[2] W. Zakowicz, J. of Apl. Phys. 55 (1984) 3421.

[3] K.V. Lotov. Driver Channeling in Laser Wakefield Accelerator. Preprint Budker INP 98-40, Budker Inst. Of Nuclear Physics, Novosibirsk, 1998

[4] L.A. Vainshtein, Open resonators and waveguides, 'Sov. Radio', Moscow (1966).

[5] C. Dragone, IEEE Trans. on Microwave theory and and Techniques, V. MTT-28, N7 (1980) 704

[6] M. Xie, Laser Acceleration in Vacuum with an Open Iris-Loaded Waveguide, to be published in the Proceedings of the Particle Acceleration Conf., Vancouver, 12-16 May, 1997, p. 660 